\title{
Latinoamérica y la educación superior en la encrucijada de la sociedad del conocimiento. Desafíos y disrupciones
}

\author{
Miguel Zapata Ross \\ Universidad de Murcia - Universidad Alcalá de Henares \\ E-mail:mzapata@um.es
}

La educación abierta en tanto superación de las dificultades, de las condiciones y de la organización para el aprendizaje, se ha constituido en un objetivo para los sistemas educativos en las sociedades desarrolladas. Las instituciones y los centros de investigación se esmeran en indagar qué modalidades de organización- son más eficaces al momento de obtener los resultados propuestos para cada modalidad de estudio o de rendimiento profesional. Se trata de ofrecer a los profesores y gestores docentes de la educación superior (ES) modalidades de diseño instruccional que consigan estos objetivos, que faciliten, en definitiva, la itinerancia de las situaciones actuales a una enseñanza universitaria abierta. Este es el desafío también en Latinoamérica. Esta editorial, si bien sirve de introducción al presente número especial de la revista Virtualidad, educación y ciencia, también intenta describir una panorámica general sobre la situación de la enseñanza abierta en línea, las tendencias y las causas que la determinan.

Se asiste a un cambio global que viene impuesto por la permeabilización del espacio interuniversitario y la emergencia de universidades globales. La disrupción universitaria, entonces, supone un reto. La forma de afrontarlo es por medio de las fortalezas intrínsecas a las universidades locales, creando iniciativas de personalización y de inclusión genuinas para las comunidades latinoamericanas y de polos de investigación propios, incluyendo los de investigación educativa, con los nuevos entornos y metodologías, con la ayuda pedagógica in situ y personalizada (Christensen en Zapata-Ros, 2013d). Es de esta forma, con este tipo de hábitats, con lo que no pueden competir las agencias internacionales.

Desde la aparición de las modalidades de educación a distancia, la universidad -o mejor cabría decir las universidades latinoamericanas- se han visto inmersas en una cultura de la diferenciación en cuanto a modalidades de enseñanza, de atención a los alumnos y, por añadidura, de atribución de perfiles diferenciados a los profesores.

Sin embargo, la sociedad del conocimiento, en los últimos tiempos, ha propiciado esquemas de convergencia. La justificación de la educación a distancia, en sus inicios, representó la superación de las dificultades y de las limitaciones que imponían las barreras de la ubicación física o de las comunicaciones. Hoy, esa razón que dio lugar al surgimiento de la modalidad de educación en cuestión, ha quedado subsumida como un obstáculo más junto con otros que pueden ser superados con las redes y la tecnología. Son las dificultades impuestas por la ubicación, pero también por la disposición de tiempo y recursos, por las diferencias etarias o culturales, o incluso, por las dificultades que impone la propia institución y su organización de la enseñanza. Como por ejemplo, una misma atención para todos los alumnos, independientemente de cual sea su perfil o su situación de aprendizaje. Son las barreras y las dificultades las que plantean los estándares educativos a los alumnos. Dificultades 
que, por ser propias de la sociedad imperante -la industrial-, se han aceptado como inevitables. Por otra parte, se constata el papel que ha jugado y está jugando la tecnología en la superación de estas dificultades. Ahora bien, la misma tecnología que ayudó al surgimiento y la evolución de la educación a distancia convencional, puede ser útil al desarrollo de todas las modalidades de educación, y a su amparo se han producido innovaciones docentes que antes eran impensables.

De esta forma, la educación abierta -en tanto superación de todas estas dificultades- se ha constituido en un objetivo para los sistemas educativos en las sociedades desarrolladas. Y sus instituciones y centros de investigación orientan sus esfuerzos en indagar qué modos de organización, con usos más adecuados de aplicaciones -affordances- son más eficaces para obtener los resultados de aprendizaje propuestos para cada modalidad de estudio y un rendimiento profesional óptimo. Se trata de ofrecer a los profesores y gestores docentes de la enseñanza superior, modalidades de diseño instruccional que consigan estos objetivos y que faciliten, en definitiva, la itinerancia de las situaciones actuales a una enseñanza universitaria abierta.

El presente trabajo sirve pues a un doble objetivo: presentar y atribuir sentido al número especial de la revista Virtualidad, educación y ciencia y, por otro lado, intentar realizar una panorámica general respecto de la situación actual de la enseñanza abierta en línea, las tendencias en presencia y las causas que la determinan.

\section{El contexto}

En el albor de la Edad Moderna, las universidades (en su primera configuración) nacen y se generalizan en Europa y en la América Hispana; este fenómeno se produce por la confluencia de causas que son exógenas: la invención de la imprenta y la necesidad de difundir y hacer útiles los conocimientos (hasta entonces preservados en abadías y cenobios, desde la época del mundo clásico y durante toda la Edad Media) y de ponerlos al servicio de las elites dirigentes e intelectuales. En ese contexto se produce una transformación fundamental: aparece el libro impreso, y eso supone un cambio radical en la forma en cómo se pone a circular el conocimiento. Pero lo sustancial, es que esos conocimientos conservados se integran a los nuevos soportes y procedimientos de diseminación; conocimientos que se habían impartido en las primeras universidades, en plena Edad Media, mediante unos rudimentos docentes surgidos en los cenobios. Entonces, la modalidad heredada se integra a las instituciones emergentes, no se opone de ningún modo a lo nuevo sino que, por el contrario, encuentra un espacio incomparablemente más amplio donde expandirse: si bien el libro sustituye a los códices, la cátedra es muy parecida al púlpito.

Cuando aparecieron la mecánica cuántica o la teoría de la relatividad, nadie las contrapuso con la mecánica clásica de Newton y de Leibnitz, sino que estas quedaron integradas como restricción de otras teorías más generales que continuaron siendo válidas en entornos locales. No debiera suceder algo distinto con las investigaciones y con los desarrollos teóricos existentes sobre aprendizaje y enseñanza, en relación con la pedagogía necesaria en los nuevos entornos. No validar su vigencia o no investigar al menos su productividad y sus variantes en los nuevos contextos, supondría una pérdida difícilmente justificable. Por el contrario, es de esperar que se produzca una continuidad en la línea de adaptación de las estrategias docentes, de la ayuda pedagógica a las nuevas condiciones. 
De esta forma, el hecho de que se abran o se liberen los contenidos del conocimiento, su representación en texto, imágenes o multimedia, no significa que automáticamente se incorporen a los esquemas cognitivos de los individuos y, mucho menos, atribuyéndoles sentido, significatividad y capacidad de ser transferidos a situaciones cambiantes o para resolver problemas. Ante la abundancia de datos e informaciones, se torna necesario dotar de instrumentos que permitan establecer enlaces entre estos recursos y el mundo de las representaciones y de las cogniciones de los alumnos. Una buena base son los conocimientos instruccionales existentes.

Como hasta ahora, pero aún más porque el medio es más complejo, habrá que establecer métodos y estrategias para adaptar los bienes del conocimiento, los OER (open educational resources) y los contenidos de los MOOC (massive open online courses) a las distintas formas de aprender, a los distintos esquemas y a las representaciones individuales; para posibilitar que los alumnos hagan sus propias elaboraciones; y para que sean transferidas en contextos diferentes, atribuyéndoles sentido. En definitiva, se trata ahora de posibilitar el aprendizaje en los nuevos contextos y con las nuevas affordances.

Ese propósito de las teorías y de las prácticas de la enseñanza tiene su tradición en el diseño instruccional, de hecho constituye su esencia. No es nuevo, arrancó -como hemos dicho- en los estudios de Reigeluth, que le llevaron a formular, en sus distintas versiones, la Teoría de la elaboración (Reigeluth et al, 1994). Esta teoría tiene su origen y su fundamentación en los trabajos previos de Ausubel, Gagné, Merril y Novak y continúa con las teorías y técnicas del diseño instruccional (Reigeluth, 2012). Constituye, como hemos visto, el entramado teórico del que se nutre el diseño de los MOOC, como caso más significativo, pero también de las iniciativas más exitosas aplicadas en las affordances de los nuevos entornos, de la web social, y de la creación de entornos personales de los alumnos, así como de las posibilidades de la analítica de aprendizaje personalizado, que permiten diseños de programas formativos centrados en los alumnos y en el aprendizaje.

Por lo tanto, la vigencia de los conocimientos, teorías, investigaciones sobre cómo se produce el aprendizaje y sobre cómo organizamos la instrucción, sería lo primero que parece que permanece en una educación universitaria de calidad.

No es nuestro objetivo aquí hacer un análisis económico o sociológico. Por lo cual vamos a prescindir de los aspectos de esta naturaleza, como analizar el modelo de negocio que va a haber detrás de la nueva ES, sea de rentabilidad social o de rentabilidad corporativa. Solo utilizaremos ese tipo de referencias cuando sea inevitable para justificar algún rasgo de la forma de aprender, por ejemplo, si un determinado recurso es sostenible o merece la pena en función de la ganancia pedagógica que produce. O cuando hagamos referencia a las causas que hacen que se produzca el abandono y mencionemos, en relación con ello, la falta de confianza en los títulos.

Centrándonos pues en ese análisis podemos detectar que existen líneas de fuerza o vectores que no sólo son una constante en todo el proceso de cambio sino que crecen y lo caracterizan: tendencias consolidadas y la eficiencia del aprendizaje como eje de modelos y procesos.

Hay ciertos rasgos que, muchas veces, pasan desapercibidos porque se tienen asumidos implícitamente o porque su origen resulta ya lejano, pero no por eso son menos importantes u 
operantes.

Uno de estos rasgos es la evolución de los recursos y entornos que están operativos en un momento. Si miramos los recursos abiertos (entendiendo por ellos no solo los recursos educativos propiamente dichos, sino la edición científica de acceso abierto y el open source) podemos constatar que todo el movimiento MOOC se produce como una evolución de los recursos abiertos, y que este nace con los objetos de aprendizaje, y continúa con los OER, hasta llegar a los cMOOC, que no son más que una forma de crear un acceso comunitario a los recursos abiertos, con un mínimo compromiso de aprendizaje y de diseño. E incluso lo hemos considerado como una subclase de la evolución del e-learning.

Pero esta tendencia no es exclusiva del ámbito educativo, es coincidente con otros fenómenos que hemos considerado básicos, específicos y definitorios de la sociedad postindustrial. Los recursos abiertos son el equivalente en la educación a lo que sucede en otros ámbitos (la música, la edición de libros, el entretenimiento, etc.). Es la bajada de precio o la gratuidad, debida a la naturaleza del procedimiento de circulación y reproducción, que se produce en la parte material de los contenidos soportados digitalmente. Lo que pone de relieve que, en el conocimiento, el valor no está en la representación material, en el ejemplar tangible o en el contenido. Estos procesos dan lugar a las innovaciones disruptivas (Christensen, 2012 y 2013) y a la napsterización ${ }^{1}$.

Como consecuencia inevitablemente ligada, los recursos serán abiertos y esto no le restará valor a la opción formativa, porque el valor va a estar en otro punto, en lo que Christensen (2012 y 2013) llama "núcleo no extensible", es decir, en la matriz, en la fuente de donde surge el conocimiento, la investigación, y en la ayuda pedagógica.

El segundo elemento lo constituye la personalización. Convendría, si hablamos de tendencias previsibles, precisar este concepto. Ya dijimos en la introducción que la palabra personalización quizá resulte confusa o ambivalente para quienes se inician en el mundo del diseño de la enseñanza y provienen de otros como el de la computación. También descartamos la expresión individualización o adaptación, porque cada una de esas palabras tiene una carga semántica por el uso en los medios especializados que no se adecúa el sentido que queremos utilizar.

La expresión personalización (en consonancia con el uso que se le ha dado en los medios interdisciplinares y por ser el término que convencionalmente se ha aceptado) hace referencia a

1 El fenómeno de la napsterización hace alusión a un programa, Napster, que adquirió mucha fama y uso hace unos años, y que permitía a los usuarios intercambiar, mediante un repositorio, música, libros, vídeos y otras obras soportadas en ficheros de ordenador. Entre nosotros fue más conocido un sucesor suyo: eMule. El fenómeno consiste en una trivialización de contenidos al prescindir de la mediación tanto de especialistas como de servicios. Según los autores que han hecho popular el término (Clark, 2012), este proceso tiene tres fases: La democratización, la descentralización y la desintermediación. Sin embargo, no acaba aquí. En -otros casos, ha posibilitado, además, la aparición de un modelo de negocio. Clark (2012) sostiene que los programas tipo Naspster y eMule allanaron el camino para el iPhone de Apple y el iPad. Y tiene razón, ya que condujo a la desintermediación en la industria musical. Es el peligro de la disrupción de la enseñanza universitaria: la desintermediación. La cuestión es cómo se producirá la napsterización del aprendizaje, y sobre todo cuáles serán los efectos en este caso de la trivialización de los contenidos de aprendizaje. 
la utilización de los recursos que la tecnología nos proporciona para, a través de su socialización, conseguir una percepción lo más completa posible respecto de cuáles son las preferencias y las representaciones que el individuo posee de la realidad y del mundo que le rodea, cuáles son las características de su andamiaje cognitivo para, a partir de él y de su rol en él, presentar los nuevos conocimientos (Hopkins, 2007). La expresión "centrado en el estudiante", según Reigeluth, se define como:

La perspectiva que empareja un enfoque en los estudiantes individualmente (...) con un enfoque en el aprendizaje (el mejor conocimiento disponible sobre el aprendizaje y cómo se produce). (ZapataRos, 2013e).

Existe una nueva línea en desarrollo: la analítica masiva de datos personalizados aplicados al campo. Son los algoritmos utilizados en otros medios y con otras herramientas, pero adecuadamente orientados a las teorías del aprendizaje personalizado por técnicas pedagógicas y de diseño instruccional. De esta forma, se pueden obtener informaciones para ajustar mejor la intervención educativa, mejorar el rendimiento de cada alumno, aumentar su satisfacción y la eficacia del programa educativo.

Otro aspecto importante es la identidad digital, el perfil de aprendizaje en la red. Se trata de utilizar los recursos que la tecnología nos proporciona para -a partir del cúmulo de datos e informaciones que se producen a partir de la socialización del alumno- obtener los rasgos relevantes en la constitución de un perfil individual con relación al aprendizaje.

Por otro lado, si está cambiando el paradigma educativo, en el sentido de que los sistemas existentes basados en la selección de los individuos sean sustituidos por otros basados en el logro personal, es coherente que se haga con el concurso de la tecnología.

También hemos visto que estas posibilidades de personalización posibles con la tecnología, pueden hacer avanzar en el horizonte señalado por el problema de dos sigmas de Bloom (1984) (Zapata-Ros, 2013).

En definitiva, se pueden cambiar los sistemas de educación y de capacitación (los aún vigentes, que fueron diseñados para seleccionar a los alumnos) por otra alternativa más deseable, en la que se produzca un cambio hacia sistemas diseñados para maximizar el aprendizaje mediante el análisis y la adaptación de las estrategias de enseñanza para no solo enfocarlas a los estudiantes que, de forma natural, sean competentes en determinadas áreas.

Esta transformación es posible hoy por los avances de la tecnología educativa, pero también por los desarrollos teóricos (en parte, desconocidos por el gran público y por buena parte de las autoridades, los gestores y los técnicos) respecto de estudiar los casos individuales para determinar las formas, las metodologías, en las cuales la instrucción debe diferir de unos a otros, agrupando a los alumnos en función de variables como son las similitudes de perfiles de aprendizaje y los rasgos de competencias.

Este sería pues el horizonte deseable frente a los desafíos que nos plantean las disrupciones en la sociedad del conocimiento y, en particular, en Latinoamérica. 


\section{Un dilema}

En lo siguiente, abordaremos el dilema ante el cual se encuentra la evolución de la ES en Latinoamérica:

- Promover la personalización y la inclusión pedagógicas empoderadas por la tecnología

- o alentar el reclutamiento de talentos.

Existe una línea de pensamiento sobre la universidad, sus funciones y su misión que ha permanecido invariable desde sus inicios y que, lamentablemente hemos constatado, es común en Latinoamérica. Es un pensamiento que satisface las expectativas de muchos profesores, gestores y de no pocos alumnos universitarios y sus familias.

Para esta posición, existen fórmulas dentro de la educación que establecen vínculo -solo a través de los materiales con contenidos- entre especialistas destacados en determinadas materias y un tipo de alumno aventajado para adquirir el conocimiento que, claramente, constituye una minoría. En este sentido, la educación se hace con materiales expuestos y organizados mediante explicaciones de tipo conferencial a un auditorio masivo y con exámenes de pruebas objetivas. Esa sería la fórmula correcta de la enseñanza universitaria y, para muchos, la única, ya que lo que no está contemplado en ese método de transmisión del conocimiento no es educación.

Estas fórmulas siempre han asegurado el éxito y han dado satisfacciones a un modelo de profesor. Pero han dejado afuera una gran cantidad de alumnos y, por ende, de recursos humanos. Son los alumnos quienes están en la parte central de la campana de Gauss.

La sociedad hasta ahora ha conferido a la universidad la misión de seleccionar a esa élite, y esa misión incluso sigue vigente en algunas modalidades disruptivas como los MOOC. Sin embargo, las sociedades, y más aún las sociedades en desarrollo, no pueden prescindir de los recursos que permitan desarrollar las capacidades profesionales o de creación de conocimiento de los alumnos, y esos, según el problema de dos sigmas de Bloom (1984), son casi todos. Tampoco se pueden desatender las expectativas personales: las situaciones de frustración no deben estar en el horizonte del bienestar que toda sociedad debe perseguir para sus miembros.

Esta línea, la de la educación de clases expositivas con exámenes, va a continuar; es la esencia de los xMOOC y de las opciones disruptivas de ES como los nanodegrees, los micromaster, y otras propuestas similares que, una vez transformadas en una modalidad de formación universitaria (Alianza OEA), apuntan al objetivo que señala Ferenstein (2014):

- hay muchos estudiantes brillantes de todo el mundo que sólo tienen acceso a los cursos a través de Udacity o KhanAcademy; la Alianza OEA permitiría a estos prodigios, por primera vez, para demostrar su talento a las empresas de tecnología, tomar cursos y obtener certificados de los proveedores de educación en línea.

- La OEA va a crear (esperemos) una nueva meritocracia en la educación superior. 
Pero en este dilema, hay otra línea de desarrollo en relación con el uso de la tecnología como apoyo a la educación universitaria: es lo que se conoce como línea adaptativa ${ }^{2}$ o de personalización.

Optamos por este último término ya que se ajusta mejor al sentido que queremos transmitir. La personalización hace referencia a la utilización de los recursos que la tecnología nos proporciona para, a través de su socialización, obtener una percepción lo más completa posible de las preferencias y las representaciones que el individuo posee de la realidad y del mundo que lo rodea, identificar cuáles son las características de su andamiaje cognitivo para, a partir de él y de su rol en el proceso, presentar los nuevos conocimientos. Es lo que sucede, con otro fin, con los algoritmos de recomendación en las búsquedas personalizadas: utilizan una gran cantidad de información procedente solo del individuo, y de las referencias anteriores que poseen de este para orientar la búsqueda. En el caso de la analítica del aprendizaje, el proceso va gobernado por la información que se obtiene del individuo a través de su actividad, tanto del procesamiento de la información que hace en su elaboración como de su mundo de relaciones sociales, sin el cual no se produciría con igual eficiencia lo anterior, o simplemente no sería posible.

Hay una corriente que apoya esta evolución. La transformación que estos autores plantean para la educación tiene su origen en los cambios sociales (y de todo tipo) que se sitúan en el pasaje de la era industrial a la era del conocimiento y, subsiguientemente, entre dos etapas muy diferenciadas del desarrollo de las teorías del aprendizaje. La primera, donde las teorías están orientadas para describir y clasificar a los alumnos desde la perspectiva de sus capacidades, y la segunda, caracterizada por las teorías que apuntan a maximizar el aprendizaje de todos los alumnos. En una, el progreso se mide en función del tiempo (pautas y ciclos temporales: cursos, clases, etc.); en la otra, se basa en los logros individuales (Reigeluth, 2012).

Por otra parte, nunca como ahora ha sido posible intervenir en la evaluación de los procesos de aprendizaje y adaptar la ayuda pedagógica a los estilos singulares a partir del análisis de lo que se ha dado en llamar la huella digital de aprendizaje, desde la analítica del aprendizaje.

2 Descartamos la expresión "adaptativo" por dos razones. La primera para diferenciarlo del "aprendizaje adaptativo", suficientemente conocido, que únicamente utiliza las necesidades de aprendizaje del estudiante para presentarle los contenidos de aprendizaje, y en nuestra propuesta, lo que se individualiza es el diseño instruccional en su conjunto, para adaptarlo a las características de aprendizaje detectadas en el entorno personal del alumno. Y la segunda para hacer más énfasis en el sujeto de la expresión, que es el que tiene que hacer el esfuerzo para adaptarse a una situación compleja y cambiante. El ser humano es, en un plazo más largo que el de su vida, un sistema adaptativo. Pero nosotros hablamos de sistema educativo. Aquí, la fuerza recae sobre el objeto del sistema, en este caso, el individuo y sus características de aprendizaje. La expresión adaptativo se vincula con sistema adaptativo complejo (CAS, por su sigla en inglés complex adaptive system) que es un tipo especial de sistema complejo. El ser humano sí se puede considerar como un sistema complejo, como lo es la bolsa de valores o un ecosistema, en el sentido que se utiliza en estos dominios teóricos. En el sentido de que es diverso y conformado por múltiples elementos interconectados, algunos de los cuales son emergentes. Pero, en este caso, los sistemas educativos no hacen énfasis en estos aspectos o en esta dimensión del ser humano por ser una dimensión ontológica que no tiene como referencia al individuo singular. A los sistemas educativos les interesa el individuo singularizado en un intervalo de tiempo que afecta a una fase de su vida o exclusivamente a su vida. En todo caso, cabría hablar de meta-adaptativo pero solo atendiendo a las formación de las competencias que desarrollan características adaptativas del individuo. 
Esta tendencia supone una línea de continuidad en la adaptación de las estrategias docentes, de la ayuda pedagógica, acercando los contenidos a las distintas formas de aprender y de integrar los conocimientos en los esquemas y en las representaciones individuales. Como vimos las aportaciones más relevantes proceden de los trabajos que hace Reigeluth, de las distintas versiones de la teoría de la elaboración, y de los trabajos previos sobre el andamiaje cognitivo, la significación de los contenidos de aprendizaje, los esquemas cognitivos, y las distintas teorías de secuenciación que realizaron Ausubel, Gagné, Merril y Novak. A la luz de estas teorías, continúan en la definición de técnicas del diseño instruccional que permitan confeccionar programas formativos centrados en los alumnos y en el aprendizaje. Por último, esa línea culmina, por ahora, en investigaciones y desarrollos prácticos para transformar los sistemas de educación y de capacitación vigentes.

Esto también es posible debido a los avances de la teoría y de la tecnología instruccional que permiten observar las situaciones que determinan cómo la instrucción debe diferir de unos casos a otros en función de variables tales como las similitudes y los agrupamientos por perfiles individuales.

Otra evolución a partir de la experiencia de los MOOC es la del reclutamiento de talentos.

Podemos señalar, a título de ejemplo, el caso de Udacity, uno de los tres oligopolios del complejo: universidades de excelencia - empresas tecnológicas - agencias MOOC (u otras disrupciones universitarias), que tiene como objetivo para la OEA, como citamos anteriormente, "seleccionar los estudiantes más brillantes de todo el mundo" (Ferenstein, 2013).

Esta es pues la alternativa, si bien no contrapuesta ni excluyente a la anterior. Solo que la personalización está lejos de ser considerada como un modelo válido por Udacity y, suponemos, por otras agencias.

Esto tiene implicaciones de coste y, en consecuencia, metodológicas. Las grandes agencias no ven interés en invertir en alumnos que precisan ayudas económicas, si pueden obtener talentos a bajo coste. Así pues, en este caso, en los MOOC y sus derivaciones disruptivas, se trata de establecer un marco donde, en un contexto de acceso autónomo a recursos, explicaciones enlatadas, etc., sobrevivan aquellos alumnos más dotados, con competencias naturales para la materia (talentos) o con más capacidad de aprendizaje autónomo, sin preocuparse por los demás.

\section{La evolución de la educación universitaria a partir de los MOOC y de otras innovaciones disruptivas en América Latina}

Hasta aquí el análisis es indiferenciado. Podría aplicarse a entornos, instituciones o sistemas de ES tanto en Latinoamérica, Europa, EE.UU o en extremo Oriente Asiático. A continuación, nos plantearemos las mismas cuestiones en relación con la evolución de la ES en las sociedades y países que se pueden considerar, desde la perspectiva de la OEA, como países destinatarios. Para ello, tomaremos como referencia, por ser el objetivo del artículo (y del número monográfico), los de América Latina.

De esta forma, nos preguntamos: ¿cuál sería la repercusión en las universidades de estos países? ¿Cómo afectarán las propuestas disruptivas, herederas de los xMOOC y sus propuestas didácticas a 
las carreras, a las facultades, a las cátedras, etc.?

En principio, no habría por qué imaginar lo que pasará en América Latina si se adopta el modelo MOOC para sus carreras oficiales universitarias, es decir, para sus estudios acreditados. No hay por qué imaginarlo porque de hecho esto no se ha producido de forma significativa, ni estable, ni sostenible en ningún lugar. Otra cuestión son los productos educativos que de ellos se derivan: nanodegrees, micromasters, credentials, etc. En trabajosos anteriores (Zapata-Ros, 2014), hemos reseñado casos notables. Por ejemplo, en la Universidad estatal de San José se hizo la experiencia (Udacity) y tuvo que deshacerse por presiones de los propios profesores. En algunos casos, como en las universidades de Coursera, lo máximo que llegan es a acreditar en el modelo oficial a alumnos procedentes de los MOOC. Pero, sobre todo, el caso que más ha avanzado en esta línea es el de Udacity, con el master del Georgia Tech que es la evolución de los xMOOC a partir del modelo rechazado en su configuración actual por Thrun en su entrevista y declaraciones a la revista Fast Company; y lo han reconvertido en master. Otro tanto sucede con los cMOOC y con los xMOOC de EDX-Fundación Belinda y Bill Gates, tras la adscripción de Siemens a esta organización. En este caso, adosan un MOOC a un master con pasarelas.

Se ha demostrado pues que no es viable en los cursos desarrollados por estas agencias por la imposibilidad de integrar -en un marco de gestión académica estándar, con diseño instruccional, evaluación, acreditación, etc.-, un sistema que no es evaluativo, que apenas tiene interacción profesoralumno, y en especial, que no satisface requisitos mínimos de eficiencia de algún tipo de aprendizaje, como ya hemos señalado en relación con el aprendizaje divergente (Zapata-Ros, 2013c).

Si esto es así en los ámbitos que son los genuinos, donde han nacido y se han desarrollado los MOOC, ¿qué se puede esperar que suceda en universidades donde los tendrían que adoptar como algo no propio y con estructuras más enclavadas en esquemas de promoción social como son los casos de América Latina? Mencionamos la promoción social porque es el factor que más los ha potenciado en EE.UU. y en Europa, junto con el de excelencia.

Igualmente, nos podríamos plantear cuáles serían los impactos que generaría esta inclusión metodológica sobre la educación convencional y en los cursos universitarios aun de corte tradicional.

A este respecto, el impacto no se va a producir de facto porque, consistentemente con lo dicho en el apartado anterior, no se van a incluir. Pero sí va a haber un impacto, y debe de haberlo, porque lamentablemente en las universidades latinoamericanas, los gestores de docencia y currículum adolecen de mímesis. Los MOOC han generado una tendencia que contribuyó a prestigiar, a la docencia virtual en general. De hecho hasta ahora se dudaba bastante de la eficiencia en cuanto a la adquisición de ciertas habilidades, sobre todo prácticas, no solo de la educación a distancia, sino también de la educación a distancia tecnológica y de la educación en entornos virtuales. Y es precisamente ahora cuando las universidades de prestigio (Harvard, MIT, Princenton, Edimburgo, etc.) han implementado los MOOC como una operación de marketing, cuando las autoridades y el público han vuelto la mirada sobre las modalidades virtuales.

Por tanto, el impacto más notable va a ser la dignificación y el crédito de las modalidades de docencia virtual universitaria. 
En la fase actual, de escaso desarrollo, con muy pocos estudios y experiencias en grado o posgrado no profesional, no sabemos cuál puede ser el nivel de aceptación por parte de las nuevas generaciones de estudiantes a tiempo completo (otra cosa son los profesionales en servicio) ante este tipo de propuestas educativas. Pero, indudablemente, los MOOC están produciendo un impacto sobre la población potencialmente universitaria en Latinoamérica. No por favorecer una mayor igualdad, que es lo que según la publicidad se va a producir, sino por ejercer un atractivo y proporcionar un canal de integración en los estudios universitarios y, particularmente, en universidades de prestigio, a los individuos talentosos de ese sector de la población estudiantil oriunda.

Hace tres años (Zapata-Ros, 2014) decíamos que la estructura híbrida de algunos master que llevan un xMOOC adosado, permite un flujo entre las dos estructuras, y posibilita que estos alumnos puedan ser captados. Ahora las estructuras de rendimiento (nanodegrees, micromaster, credentials, etc.) permiten hacerlo directamente. Esto supone un peligro que las universidades y los gobiernos latinoamericanos deben afrontar. Y deben hacerlo cambiando en profundidad la naturaleza y estructura de su oferta de estudios. Pero no solo cambiándolo en aspectos tecnológicos (este es el error, la percepción trivial del asunto), sino hacerlo con sistemas, métodos y talantes que permitan una ayuda personalizada y tutorizada. Un cambio de la naturaleza que describimos, que permita a este tipo de alumnos, los talentosos, integrarse en una educación superior de calidad, y satisfactoria para sus expectativas, en su propia cultura de origen.

De esta forma, si bien descartamos una sustitución aún parcial de los estudios universitarios reglados por alguna modalidad de MOOC, sí que es previsible que las modalidades de disrupciones universitarias de rendimiento emergentes desplacen, en el hábitat de los estudios superiores latinoamericanos, un número mayor o menor de las propuestas existentes, sobre todo a las que no se adapten, como ha sucedido en otros ámbitos de las actividades productivas o de los servicios: las finanzas, los medios de comunicación, el mundo editorial, o el comercio y distribución de mercancías.

Ante esa eventualidad y aceptando las modalidades innovadoras que utilizan la tecnología para una mayor personalización y para la consecución de aprendizajes más eficientes (ya que incluyen aspectos como los que hemos considerado: son abiertos, personalizados, situados, etc.), podríamos plantearnos, igualmente, cuáles deberían ser los rasgos más sobresalientes de los actores implicados para conseguir esos progresos.

Y puestos a ello, en primer lugar, están los profesores.

Un rasgo que poseen, en general, los profesionales es la conciencia de pertenecer a un estamento. Según las situaciones o sociedades, esta conciencia se da en mayor o menor grado. A lo largo de estancias en distintos países y regiones latinoamericanas, de años de trabajo y de realización de proyectos e investigaciones en comunidad, de contacto con especialistas y de lecturas de trabajos, tesis, artículos y proyectos, podemos afimar que existe un rasgo común en los profesores universitarios de Latinoamérica: es el alto grado de conciencia estamental. Pues bien, para una inculturación en estas nuevas modalidades de educación es necesario que esta conciencia no sea un obstáculo a la hora de asumir los cambios que se deben producir. Los atributos de casta y los estereotipos sociales y gremiales son enemigos de este cambio. En esencia, constituyen una causa de prejuicios conservadores. 
Así, el profesor debe ser receptivo al trabajo en los nuevos entornos y con los nuevos recursos. Debe ser un profesor abierto a los nuevos métodos: que utilice el mastery learning, que fomente estrategias metacognitivas entre sus alumnos y, como leit motiv de sus actividades, fomente estrategias de trabajo autónomo, colaborativo, intercultural, de clase invertida (flipped classroom), etc. Debe utilizar el diseño instruccional, la evaluación y la investigación formativa o basadas en el diseño. También, el diseño basado en competencias y en resolución de problemas.

En definitiva, se busca un perfil de docente que sea flexible y que presente una excelente disposición a adoptar los cambios que estos nuevos entornos suponen.

Para el análisis que estamos haciendo tenemos en cuenta la situación de progreso y las tendencias presentes en la educación abierta en línea. El esquema de progresión que sigue lo iniciamos en 2015 (Zapata-Ros, 2015), continuó con motivo de los trabajos sobre smart universities (Zapata-Ros, 2016) y lo hemos actualizado en función de este trabajo a partir de MOOC Trends in 2016: College Credit, Credentials, and Degrees (Shah, 2016): ${ }^{3}$

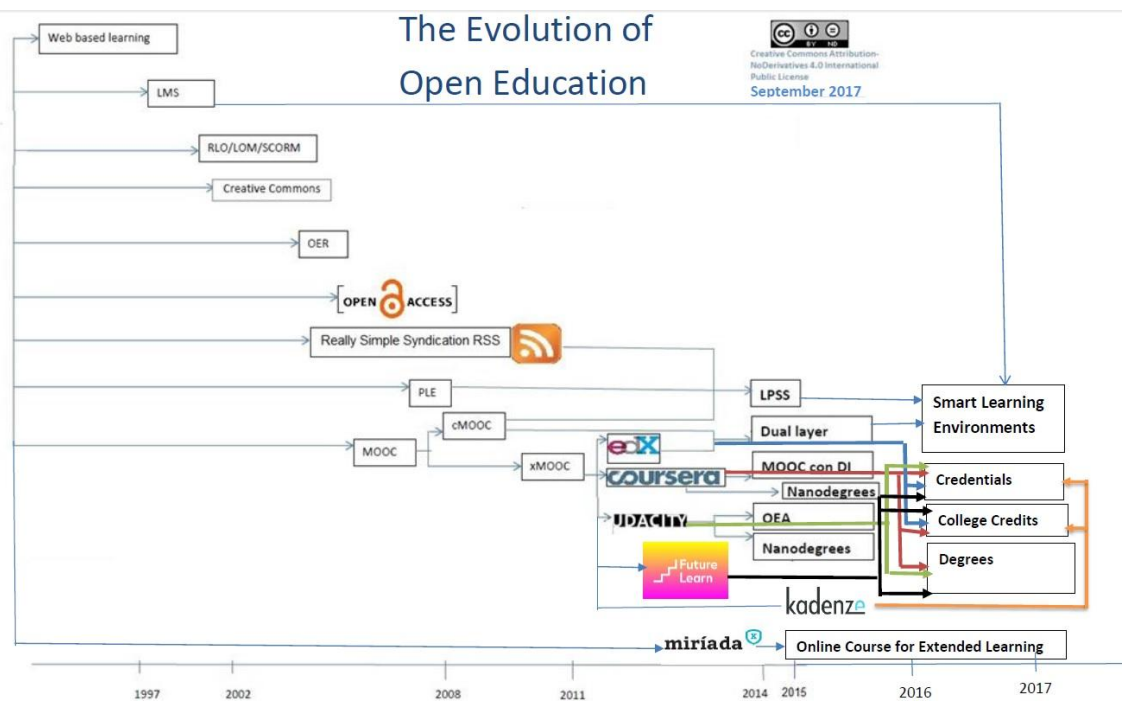

Fuente: Elaboración propia

Respecto a la repercusión que posiblemente tengan estos procesos educativos globales en materia de evaluación y acreditación, una cuestión han sido los MOOC, cuyo recorrido ha concluido en el área donde se generaron: EE.UU. y Canadá; y otra, los entornos y las configuraciones de amplios sectores o ámbitos de la educación universitaria que han quedado como resultado del cambio que se

3 Para actualizarla se han utilizado, además de las referencias de este documento, la serie de posts que, a partir de junio de 2014 (Zapata-Ros,) están publicados en Tumblr, en redes abiertas y en RED de Hypotheses con el título genérico de "Los MOOC han muerto"(2016) y de los enlaces que de ellos se derivan. Para las últimas actualizaciones de la evolución de las disrupciones, hemos utilizado el artículo "MOOC Trends in 2016: College Credit, Credentials, and Degrees" (Shah, 2016) y los documentos enlazados. Todos ellos se sugieren a los interesados investigadores y estudiosos de este tema. 
está produciendo, del cual un síntoma (no el más relevante ni el más profundo) han sido los MOOC.

Los cursos masivos por sí mismos no suscitarán ninguna transformación notable. Pero el cambio que se está produciendo en la ES, y que tiene como rasgo más visible a los entornos basados en la tecnología, sí se presume profundo e irreversible. Afecta a los aspectos más centrales como el rol que desempeña la clase y el trabajo autónomo de los alumnos (en la lipped classroom, por ejemplo). Afecta a la evaluación, al papel nodal que en ella jugará la analítica de aprendizaje, es decir, el análisis de todo lo que el alumno produce en su entorno de trabajo, a su naturaleza de material vivo para evaluar su progreso y el del propio sistema de enseñanza. Incluso afecta a la gestión, la organización y a la configuración de cosas tales como los edificios y los equipamientos universitarios (Zapata-Ros, 2014).

El otro gran cambio, el global, viene impuesto por la permeabilización del espacio interuniversitario y la presencia de universidades globales. La disrupción universitaria supone un reto. La forma de afrontarlo es con las fortalezas intrínsecas de las universidades locales, con su "núcleo no extensible", en palabras de Christensen (2013):

En este caso ese núcleo no extensible pasaría por crear polos de investigación propios, incluyendo los de investigación educativa con los nuevos entornos y metodologías, con la ayuda pedagógica in situ y personalizada. Es de esta forma con este tipo de hábitats con los que no pueden competir las agencias internacionales. (en Zapata Ros, 2013d).

\section{Referencias bibliográficas}

ALEXANDER, B. (2008). Web 2.0 and Emergent Multiliteracies. Theory into Practice, 47(2), 150-160.

ARMSTRONG, J. y FRANKLIN, T. (September 2008). A review of current and developing international practice in the use of social networking (Web 2.0) in higher education. Franklin Consulting. Recuperado de: http://www.franklin-consulting.co.uk/LinkedDocuments/the $\% 20$ use $\% 20$ of $\% 20$ social $\% 20$ networking $\% 20$ in\%20HE.pdf [01/08/14].

BATES, T. (2014). Learning theories and online learning. Recuperado de: http:/ /www.tonybates.ca/2014/07/29/ learning-theories-and-online-learning/ [01/08/14].

BLOOM, B. (1984). The 2 Sigma Problem: The Search for Methods of Group Instruction as effective as Oneto-One Tutoring, Educational Researcher, 13(6,) 4-16. Recuperado de: http://www.comp.dit.ie/dgordon/ Courses/ILT/ILT0004/TheTwoSigmaProblem.pdf

BRANDMAN, R. (2013) 5 Tips: Learn more effectively in class with Mastery Learning. Course Operations MOOC Pedagogy Specialist, Recuperado de: http://blog.coursera.org/post/50352075945/5-tips-learn-moreeffectively-in-class-with-mastery [09/06/13]

BYRAM, M. (1992), Culture et éducation en langue étrangère, Paris, Hatier/Didier collection «Langues et apprentissage des langues».

BYRAM, M., GRIBKOVA, B. y STARKEY, H. (2002), Developing The Intercultural Dimension In Language Teaching. A Practical Introduction For Teachers, Council of Europe, Strasbourg. Recuperado de: http://lrc. cornell.edu/director/intercultural.pdf [29/05/2014] 
CHRISTENSEN, C. M. (2012) Disruptive innovation. Recuperado de: http://www.christenseninstitute.org/ key-concepts/disruptive-innovation-2/ [01/08/14].

CHRISTENSEN, C. M. (2013). The innovator's dilemma: when new technologies cause great firms to fail. Harvard Business Review Press.

CIT (Center for Intructional Technologie). (2013). Building a Coursera Course Version 2.0 https://docs.google. com/document/d/1ST44i6fjoaRHvs5IWYXqJbiI31muJii_iqeJ_y1pxG0/edit?pli=1

CLARK, D. (2012). Napsterisation of learning: Democratisation, decentralisation and disintermediation of learning. Blog Donald Clark Plan B http://donaldclarkplanb.blogspot.com.es/2012/10/napsterisation-oflearning.html

CONOLE, G. (2013). MOOCs as disruptive technologies: strategies for enhancing the learner experience and quality of MOOCs. RED, Revista de Educación a Distancia. (33). Recuperado de: http://www.um.es/ead/ $\mathrm{red} / 33$

COURSERA (2013) The 5 tips learn more effectively in class with mastery learning. Recuperado de: http:// blog.coursera.org/post/50352075945/5-tips-learn-more-effectively-in-class-with-mastery

FERENSTEIN, G. (2013). Tech Titans And Online Education Orgs Team Up With The Open Education Alliance. Recuperado de: http://techcrunch.com/2013/09/09/tech-titans-and-online-education-orgs-teamup-for-career-standards-alliance/

FERENSTEIN, G. (2014). AT\&T and Udacitypartner to createthe 'nanodegree', a new type of college degree. Recuperado de: http://venturebeat.com/2014/06/16/att-and-udacity-partner-to-create-the-nanodegree-anew-type-of-college-degree/

HOPKINS, D. (2007). Every School a Great School: Realizing the Potential of System Leadership.Maidenhead: McGraw Hill.

HARDESTY, L. (March 6, 2013). Higher-ed leaders meet to discuss future of online education MIT News http:// news.mit.edu/2013/edx-summit-0306

MCCOMBS, B., y WHISLER, J. S. (1997). The learner-centered classroom and school: Strategies for increasing student motivation and achievement. San Francisco: Jossey-Bass Publishers.

SHAH, D. (2016). MOOC Trends in 2016: College Credit, Credentials, and Degrees. Recuperado de: https:/ / www.class-central.com/report/mooc-trends-credit-credentials-degrees/

REIGELUTH, C. M. (1994). The imperative for systemic change. In C. M. Reigeluth \& R. J. Garfinkle (Eds.), Systemic change in education, 3-11. Englewood Cliffs, NJ: Educational Technology Publications.

REIGELUTH, C. (2012). Teoría instruccional y tecnología para el nuevo paradigma de la educación. RED, Revista de Educación a Distancia, (32). 30 de septiembre de 2012. Recuperado de: http://www.um.es/ead/ $\mathrm{red} / 32[01 / 08 / 14]$.

REIGELUTH, C.M., WATSON, W.R., WATSON, S.L., DUT'TA, P., CHEN, Z., y POWELL, N.D.P. (2008). Roles for technology in the information-age paradigm of education: Learning Management Systems. Educational Technology, 48(6), 32-39. 
WELLER, M. (2013). Coursera's new plans - The Ed Techie: You can stop worrying about MOOC now. Recuperado de: http://ow.ly/1WTQCf

ZAPATA-ROS, M. (2013). El “problema de las dos sigmas” y el aprendizaje ayudado por la tecnología en la Educación Universitaria. Recuperado de: http://eprints.rclis.org/18866/8/MOOCs.pdf

ZAPATA-ROS, M. (2013b). Analítica de aprendizaje y personalización. CAMPUS VIRTUALES; 2(2) Recuperado de: http://www.revistacampusvirtuales.es

ZAPATA-ROS, M. (2013c). Enseñanza Universitaria en línea, MOOC y aprendizaje divergente. Preprint en Researchgate. Recuperado de: https://www.researchgate.net/profile/Miguel_Zapata-Ros/ publication/235955610_Ensenanza_Universitaria_en_linea_MOOC_y_aprendizaje_divergente/ links/00b7d514b8ce72a982000000.pdf

ZAPATA-ROS, M. (2013d). Los MOOCs, génesis, evolución y alternativa. Génesis (I). La crisis de la universidad como legitimadora social del conocimiento. Recuperado de: http://red.hypotheses.org/505

ZAPATA-ROS, M. (2013e). Charles Reigeluth: la personalización del aprendizaje y el nuevo paradigma de la educación para la sociedad postindustrial del conocimiento. En M. Esteban y J. Sáez (coords.), Pensadores de ayer para problemas de hoy: teóricos de las ciencias sociales. (pp. 153-191). Barcelona: UOC Editorial.

ZAPATA-ROS, M. (2014). Los MOOC en la crisis de la Educación Universitaria: Docencia, diseño y aprendizaje. Amazon.

ZAPATA-ROS, M. (21/04/14) La configuración de espacios y de entornos físicos y tecnológicos en la nueva Enseñanza Superior (I). Redes abiertas. Recuperado de: http://redesabiertas.blogspot.com.es/2014/04/laconfiguracion-de-espacios-y-de.html

ZAPATA-ROS, M. (26/04/14) La configuración de espacios y de entornos físicos y tecnológicos en la nueva Enseñanza Superior (II). Redes abiertas. Recuperado de: http://redesabiertas.blogspot.com.es/2014/04/laconfiguracion-de-espacios-y-de_26.html

ZAPATA-ROS, M. (Junio de 2014). Los MOOCs han muerto (1). Micro posts de Miguel Zapata. Recuperado de: http://miguelzapataros.tumblr.com/post/88960768465/los-moocs-han-muerto-1

ZAPATA-ROS, M. (Febrero de 2015). La evolución de la Educación Abierta. Blog Redes abiertas. Recuperado de: http:/ /redesabiertas.blogspot.com.es/2015/02/la-evolucion-de-la-educacion-abierta.html

ZAPATA-ROS, M. (Marzo de 2015). El diseño instruccional de los MOOCs y el de los nuevos cursos abiertos personalizados. Revista de Educación a Distancia. 45(2). Recuperado de: http://www.um.es/ead/red/45/ zapata.pdf

ZAPATA-ROS, M. (2016). La universidad inteligente. RED El aprendizaje en la Sociedad del Conocimiento. Blog RED de Hypotheses. Recuperado de: https://red.hypotheses.org/904 